At its maximum development the endocœle extends for one third of the length of the egg, and at this stage organ-rudiments appear. Well-defined regions of hypoblast lie one on either side of the endocele $(L . H p)$ and one on the ventral side. The epiblast partially segregates into a region anterior to the endoccle, another on its antero-ventral aspect and a third ventral to the ventral hypoblastic band. The three hypoblastic bands extend posteriorly to form the reproductive organs, while anteriorly they develop into excretory system rudiments. The ventral epiblastic band forms the body-wall by the migration of its cells through the yolk to the periphery of the egg. The anterior epiblastic region is the nervous system rudiment and the antero-ventral region is the pharyngeal rudiment.

The endocole now undergoes marked reduction in size and the pharynx develops a large lumen. These two cavities unite and the endocele thus becomes the œesophagus. Cells from the wall of the œsophagus proliferate and form an intestinal wall enclosing most of the remaining yolk material. At hatching, all internal organs have developed into a functional state except the reproductive systems.

A fuller account is being published elsewhere.

ROY A. HARRISON

Zoology Department,

Victoria University College,

Wellington, New Zealand. Aug. 6.

\section{Coiling of Wool Induced by Certain Reagents}

DURING some investigations regarding twist in wool, some observations worth describing were made, though no explanation can be offered.

If a Merino wool fibre 4 in. long is anchored at each end and allowed to lie loosely in a horizontal position in a Petri dish in water, nothing obvious results. If the water is replaced with 1 per cent caustic soda $\left(20^{\circ} \mathrm{C}\right.$.) several phenomena may be observed. Thus: (1) within $1 \mathrm{~min}$. the original crimp disappears and the fibre appears 'lank' ; (2) it goes into about three coils per inch within 3 min.; (3) it goes into fine turns within $8 \mathrm{~min}$. ; (4) it winds up more closely, averaging 15-20 turns per inch by 15 min., after which no further action is observed; (5) the direction of the coil reverses here and there along the length of the fibre.

No similar phenomena were observed in acid solutions.

If, after immersion for $15 \mathrm{~min}$. in 1 per cent sodium hydroxide, the fibre is transferred to water or acid, the coils unwind; they reappear when the fibre is replaced in the sodium hydroxide solution. But if the period of immersion is two hours or more, the coils do not disappear in acid or water.

Human hair, produced in a relatively straight follicle, does not coil in a solution of 1 per cent sodium hydroxide; primary wool fibres, produced in relatively spiral follicles, go into large coils; secondary wool fibres, which come from coiled follicles, go into very close coils. No closer correlation has yet been made on this point. Wools of breeds other than the Merino behave similarly in all these respects but are less predictable as to behaviour.

It was found that under zero tension there was a 2-3 per cent decrease from the original length after immersion for $15 \mathrm{~min}$. in 1 per cent caustic soda. If this were prevented for $\mathbf{2 4}$ hours by anchoring the fibre in the caustic soda at its original length, it will not assume fine coils when, afterwards, the ends are moved closer together, but it goes into a few irregular large coils.

Large, irregular coils resulted from using the following solutions at $20^{\circ} \mathrm{C}$. : 1 or 3 per cent potassium cyanide, 1 per cent sodium thioglycollate, and $0 \cdot 125$ and 0.33 per cent sodium sulphide and 0.88 S.G. ammonium hydroxide. 1 per cent potassium hydroxide gave results similar to I per cent sodium hydroxide, but barium hydroxide was not effective in this respect.

Merino wool in I per cent sodium sulphide (anhydrous salt) at $20^{\circ} \mathrm{C}$. was then studied under similar conditions. In this solution the fibres immediately straighten out and remain straight for $1 \mathrm{~min}$. They assume large coils within $3 \mathrm{~min}$., and are very closely coiled in $6 \mathrm{~min}$. (if transferred to water at this stage these coils disappear but reappear when again immersed in the sulphide solution), and $10 \mathrm{~min}$. after immersion they again straighten. The fibres once more wind up into very fine coils $15 \mathrm{~min}$. after immersion and may then disintegrate. The lengths of six Leicester fibres, which at no stage have been under tension, had increased finally in 1 per cent sodium sulphide between 33 and 56 per cent, averaging 47 per cent.

The action of 1 per cent caustic soda may be due to a high $p \mathrm{H}$, and the further reactions of 1 per cent sodium sulphide may be due to subsequent reduction. Treatment of fibres with 1 per cent caustic soda for 15 min., followed by a wash in water which causes them to uncoil, and subsequent immersion in 1 per cent sodium sulphide resulted in the characteristic coiling, straightening and further coiling that has been described for 1 per cent sodium sulphide. Treatment of fibres with 1 per cent sodium sulphide until the first coiling has been completed $(6 \mathrm{~min}$.), followed by a wash in water and immersion in 1 per cent caustic soda, caused coiling similar to that previously permitted for these fibres in the 1 per cent sodium sulphide, but no subsequent straightening or further coiling as would be the case were sodium sulphide used finally instead of 1 per cent caustic soda. Treatment of fibres for 6 hours with 1 per cent caustic soda did not prevent them when afterwards immersed in 1 per cent sodium sulphide from straightening out and then coiling as described earlier. Such fibres, however, did not disintegrate and would bear weights of $0.1-0.2 \mathrm{gm}$. without undue stretching. This is in marked contrast to the eventual gelatinous nature of wool immersed in 1 per cent sodium sulphide without previous prolonged treatment with 1 per cent caustic soda. It is evident that the prolonged action of the 1 per cent caustic soda makes the wool resistant to the disintegrating action of 1 per cent sodium sulphide.

Obviously, further fundamental chemical, physical and histological studies must be completed before attempting to explain the phenomena described. The purpose of this note is to direct the attention of other workers to these facts so that they also may investigate what are indeed some very peculiar characteristics of wool.

Martin R. Freney

Australian Council for Scientific

and Industrial Research,

314 Albert Street,

East Melbourne. 